\title{
WETLAND CLASSIFICATION FOR BLACK DUCK HABITAT MANAGEMENT USING COMBINED POLARIMETRIC RADARSAT 2 AND SPOT IMAGERY
}

\author{
Wen Zhang ${ }^{1, *}$, Baoxin $\mathrm{Hu}^{1}$, and Glen Brown ${ }^{2}$ \\ ${ }^{1}$ Department of Earth and Space Science and Engineering, York University, 4700 Keele St., Toronto, ON, M3J 1P3, (wwzhang, \\ baoxin)@yorku.ca \\ ${ }^{2}$ Wildlife Research and Monitoring Section, Ontario Ministry of Natural Resources\& Forestry, Trent 10 University DNA Building, \\ 2140 East Bank Drive, Peterborough, ON K9L 0G2, glen.brown@ontario.ca
}

Commission VI, WG III/4

KEYWORDS: Wetlands, Synthetic Aperture Radar, Polarimetric data, Decomposition, Classification

\begin{abstract}
:
The black duck population has decreased significantly due to loss of its breeding habitat. Wetlands are an important feature that relates to habitat management and requires monitoring. Synthetic Aperture Radar (SAR) systems are helpful to map the wetland as the microwave signals are sensitive to water content and can be used to map surface water extent, saturated soils, and flooded vegetation. In this study, RadarSat 2 Polarimetric data is employed to map surface water and track changes in extent over the years through image thresholding and reviewed different approaches of Polarimetric decompositions for detecting flooded vegetation. Also, object-based analysis associated with beaver activity is conducted with combined multispectral SPOT satellite imagery. Results show SAR data has proven ability to improve mapping open water areas and locate flooded vegetation areas.
\end{abstract}

\section{INTRODUCTION}

American black ducks (Anas rubripes) experienced a long-term decline between the 1950s and 1990s causing the population to be reduced by more than 50\%. (Black Duck Joint Venture Management Board, 2015) It is important to locate and protect their breeding range habitat. The current framework needs better monitoring tools that have the ability to collect breeding range habitat information at both spatial and temporal scales that are appropriate for population management. Advanced remote sensing technology provides an excellent opportunity to obtain spatially extensive and temporally repeated data relevant to mapping black duck habitat. (Robertson et al., 2015, Rawat, and Kumar, 2015) The SAR (synthetic aperture Radar) imagery obtained by RADASAT is especially effective for its ability in the detection of water and flooded vegetation. Compared with vegetation canopies, water has strong specular microwave reflection that results to be appeared dark and homogeneous in the SAR imagery. Such information is useful in detecting flooded areas and classifying wetlands (Hess et al., 2003, Touzi et al., 2007, Lane and D'Amico, 2010). RADARSAT-2 is a fully Polarimetric satellite system that transmits and receives energy in all four planes (HH, VV, HV and VH), such configuration can maintain the phase that makes mapping the different scattering mechanisms within a wetland possible. (Brisco et al., 2011) The SAR backscatter can be decomposed into four types: (1) specular scattering (no return) indicating smoother surfaces; (2) rough scattering (a single bounce return) indicating rough water; (3) volume scattering (signal backscattered in multiple directions) indicating vegetation canopies; and (4) double-bounce (radar signal was deflected off by right-angled surfaces, and most energies returned) indicating vertical emergent vegetation is surrounded by a visible, smooth water surface. Flooded vegetation can also be differentiated by a combination of double- bounce and volume backscattering. (Wdowinski et al., 2008, Brisco and Touzi, 2008, Brisco et al., 2013) Despite studies have shown that the polarimetric information significantly improves the wetland vegetation characterization, the classification has only been done at pixel levels. (Ghedira et al., 2000, Parmuchi, 2002, Kaya et al., 2004, Touzi et al., 2007, Brisco et al., 2013) This is due to the nature of the SAR data appearance that makes the process of segmenting into image objects difficult. Therefore, in this study, we will investigate the data fusion using both optical and SAR imagery to classify wetlands at the object level. Different target scattering decomposition methods are studied to derive unique parameters, such as alpha angle and entropy that optimize the characterization of wetland classes and establish the relationship between remote sensing (SAR) parameters and physical indicators of the back duck habitat. These indices will be used with available long-term waterfowl data for modeling the contribution of habitat to demographic variation in black ducks. Our findings will help identify the value of remote sensing products to support standardized monitoring of change in wetland habitat and integration into the existing monitoring-management framework.

\section{STUDY AREA AND DATA SET}

The study area is located in Ontario, Canada (Figure 1). It is within the western portion of black duck breeding range with the southern area covered by deciduous forest and transitioning to the northern boreal forest. This area is selected as the existing waterfowl and brood dataset has sufficient information in providing detailed productivity and environmental information for individual wetlands. 


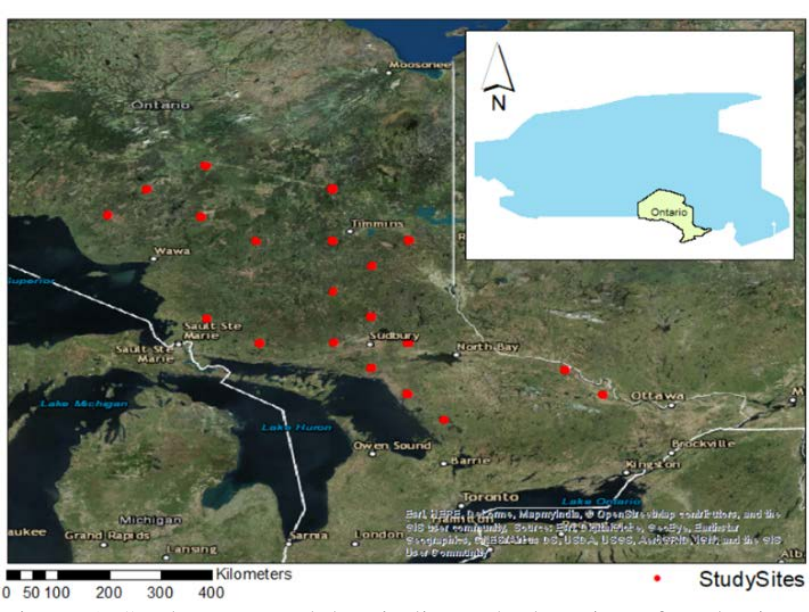

Figure 1. Study area. Red dots indicate the location of study sites.

Five Radarsat-2 SAR quad-polarization imagery acquired in May of 2008,2009,2010, 2014, 2015, and 2016, in fine resolution mode with a spatial resolution of $5 \mathrm{~m}$. Sigma Nought which is the backscatter returned to the antenna from a unit area on the ground is used to calibrate data for the local incidence angle to the ground range. All three SAR imagery was then converted from raw units to power units (decibels). The SPOT-7 imagery acquired on 03 May 2016 has four spectral bands, blue (450-520nm), green (530-590nm), red (625-695nm), and near-infrared (760-890), and a spatial resolution of 6.6 meters. The imagery has been orthorectified to be the same georeferenced as the SAR imagery.

\section{METHODOLOGY}

\subsection{SAR despeckle}

Signal-scattering in SAR data often causes image speckle or a salt-and-pepper noise. Prior to data analysis, speckle filter is used to remove high-frequency noise (speckle) while preserving edge and texture structure. In this study, Touzi filter is adapted, as this adaptive filter has a multiresolution ratio edge detector for preserving the fine structures such as the lake edges. This is effective as open water bodies are one class of our wetland classification.

\subsection{Mapping open water extent}

Mapping areas of open water have been a key component for wetland monitoring. Traditionally, the areas of water are mapped based on human visual interpretation. However, the process is very time consuming and results vary among image interpreters. NDVI and water indices calculated from optical imagery has also been major resources to be used for water mapping. This technique is dependent on weather conditions of snow and rain, and often include false positives from built-up land and saturated soil. Image thresholding from SAR imagery is the most commonly used approach to map surface water. In this method, all pixels with a backscatter coefficient lower than a specified threshold in an intensity image are mapped as water. This technique is reliable as the histogram of the image has a unique pattern of a valley followed by a Gaussian distribution, and then the bottom of the valley naturally becomes the thresholding value. To automatically chose the value, Expectation Maximization (EM) method is adapted to fit two Gaussians on the histogram, and the intersection is calculated to be the userselected threshold to map surface water. In this study, we extract the extent of water from multi-temporal RadarSat 2 despeckled image in May of 2008, 2009, 2010, 2014, 2015 and 2016.

The $\mathrm{HH}$ polarization is utilized in this study as it generally provides the best result that separate land from water. The reason account for it is due to open water results in less scattering compared to the $\mathrm{HV}$ or VV polarization and is less sensitive to capillary waves created from the wind. As a result, the differences in backscatter responses between land and water are the greatest in the $\mathrm{HH}$ polarization.

\subsection{Polarimetric decomposition.}

As the flooded vegetation interaction with the microwave radiation emitted by SAR sensors is dominated by double-bounce and volume scattering, decompose backscattered response is investigated. Many Polarimetric decomposition methods have been developed, including the Cloude-Pottier, Freeman-Durden, Touzi, general four components, Krogager, and $\mathrm{m}-\chi$ methods. Two of most used polarimetric decomposition approaches, Freeman-Durden and Touzi are reviewed, as recent studies have demonstrated their ability for mapping flooded vegetation.

The Freeman-Durden decomposition is a physically based model that estimates the amount of surface, double-bounce, and volume scattering response contributing to the total backscatter from each pixel. (Freeman and Durden, 1998) The Touzi decomposition uses a complex entity, a roll-invariant coherent scattering model for the parameterization of coherency eigenvectors used to distinguish different types of wetlands. (Touzi, 2007)

\subsection{Object-based wetland mapping}

Region growing segmentation method is employed to generate ground objects of the SPOT multispectral imagery. The segmentation parameter is determined through multiple tests to run that yield visually best wetland objects. With the objects derived from the segmentation process, the statistics of double bouncing from Polarimetric decomposition are calculated and then assigned back to the segments for wetland detections.

\section{RESULT AND DISCUSSION}

The open water map results show in Figure 2 has demonstrated that thresholding from SAR imagery was an effective method to map surface water for both large and small water bodies.

The change of surface water area from May 2008, to May 2016 are not significant. A small area of changing can be attributed to beaver activities and/or human interference. Although the threshold was automatically determined from the histogram, it worth mentioning that the selection of the threshold can introduce uncertainty into the area of water extent with the commission error taken into account with a comparison to actual environmental changes. Also, the multi-temporal analysis should keep consistent with beam mode, polarization and ancillary sources of data to minimize co-registration efforts and obtain an accurate result. 


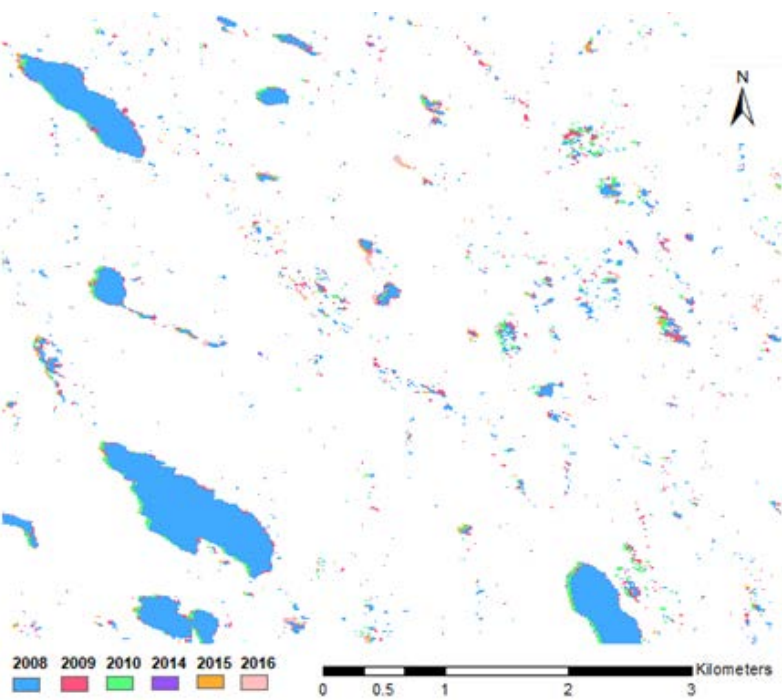

Figure 2. Open water extent map.

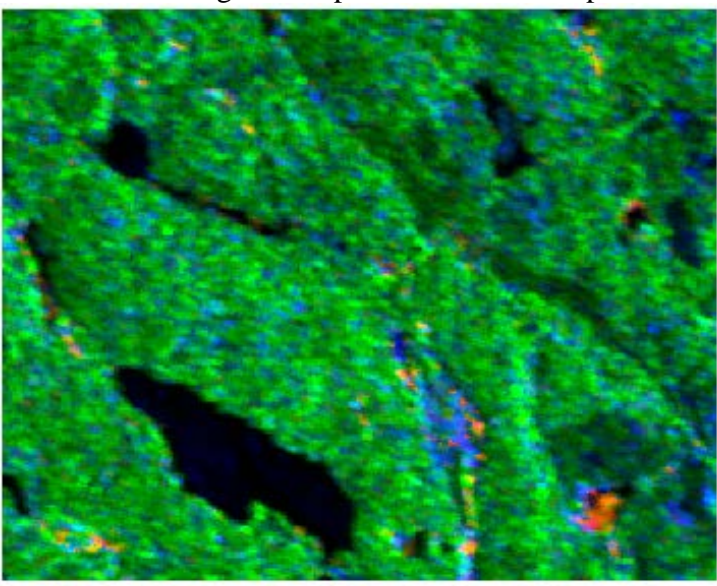

- Red: Pawer coritiauticis due ba double tiounce Green: Pawer contibutions due ba volume scatierin - Elue: Pawer corthibuforns due a rough surlaco

(a)

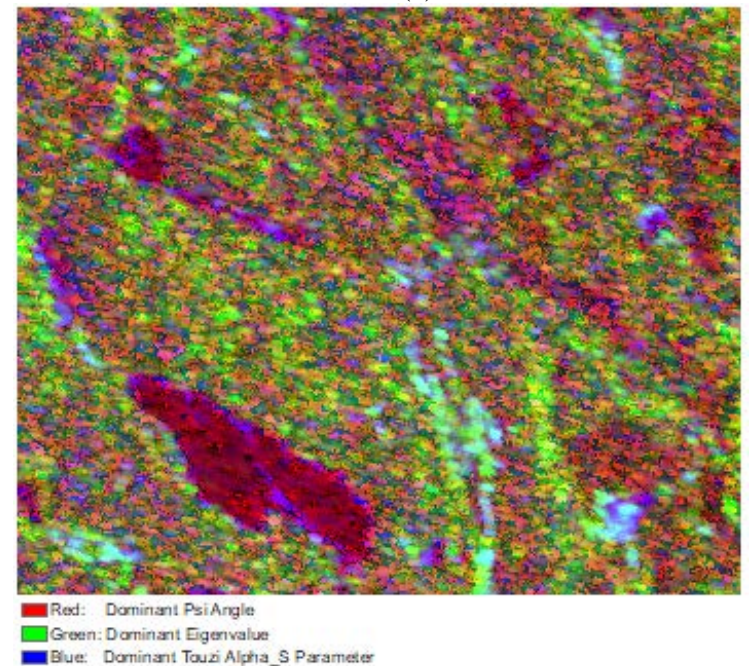

(b)

Figure 3. Polarimetric decomposition image. (a) FreemanDurden decomposition. (b) Touzi decomposition.

As shown in the Polarimetric decomposition result, Figure 3, both the Freeman-Durden and Touzi decompositions were effective for mapping changes between different land cover classes and wetland. The Freeman-Durden decomposition had a better representation for identifying lake area in this study. This is due to the reason that the flooded vegetation land cover was usually dominated by double-bounce backscatter, whereas open water had specular backscattering, upland vegetation had volume backscattering, and areas of wet soil were dominated by surface scattering. (White et al., 2015) The amount of volume and double-bounce scattering can also be an indicator of vegetation density. However, there is a problem with small, dispersed patches of flooded vegetation, as they do not return the strong double-bounce backscatter. Also the vegetation that is short or patchy can have a backscatter more similar to water, and be falsely identified. It also worth mention that the Freeman-Durden decomposition has the disadvantage to map changes from wet soil to open water as a mix of mud and vegetation can return double-bounce backscatter and creates commission errors.

Similar to the Freeman-Durden decomposition, Touzi was able to highlight wetland areas. The Touzi decomposition did not map lakes as open water clearly from the surroundings and identify the flooded vegetation as well as the Freeman-Durden decomposition. As stated in Touzi, 2007, the scattering type radiometric information provided by $\alpha$ does not effectively classify wetland. The dominated scattering type phase of the symmetric scattering type claimed to have an enhanced vegetation type discrimination that can be used to separate shrubdominated bog from sedge-dominated fen and tree bogs but can only use on coherent infarmetric data.

The flooded vegetation map is shown in Figure 4. It indicates that high probability of flooded vegetation area is coloured in orange to the yellow and low probability of flooded vegetation coloured in green to blue. The result is overlaid with beaver activity locations where star indicate present of beaver, a red circle with star indicated beaver dam, and ping circle indicated beaver lodge. As demonstrated in the result, most beaver activities are associated the flooded vegetation area, i.e. segments with high double bouncing scatter. Further investigation should perform with both ascending and descending SAR data, as the vegetation by the edge of the water (on the land) can also create double bouncing scatter and can be accidentally classified as flooded vegetation.

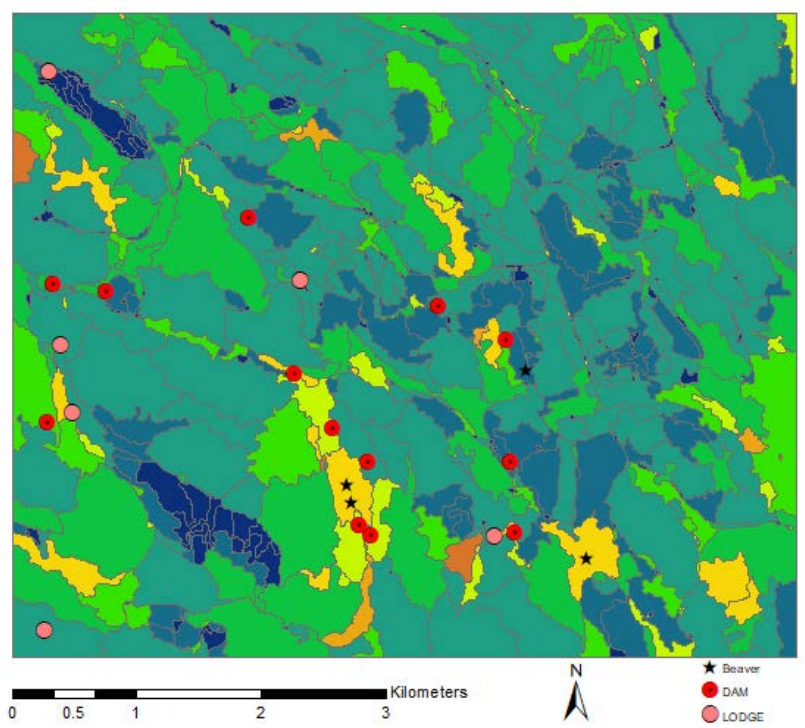

Figure 4. Flooded vegetation map with colour ranging from orange to blue, as orange colour indicates a high probability of flooded vegetation area and blue indicated low probability.

Overlaid beaver activity locations where star indicates present of beaver, a red circle with star indicated beaver dam, and ping circle indicated beaver lodge. 


\section{CONCLUSION}

The result has proven that RadarSat SAR Polarimetric decomposed image prove the ability to discriminate wetland features. Also, use of multi-sensor data, the combined Radarsat2 , and SPOT multispectral imagery was preferred over the individual RadarSat and SPOT image data for interpreting wetland extent and flooded vegetation. Segments generated from multispectral images can be easily interpreted and displayed. Object-based classifications appeared to have fewer isolated wetland pixels and wetland integrity compared to the pixelated RadarSat Polarimetric decomposition result. To better classify the wetland for black duck habitat mapping, future work includes data fusion between optical imagery and SAR Polarimetric data and machine learning object-based classification.

\section{ACKNOWLEDGMENTS}

The authors are gratefully acknowledged to Ontario Ministry of Natural Resources and Forestry for providing the RadarSat 2 data. This research was made possible by financial support Natural Sciences and Engineering Research Council (NSERC).

\section{REFERENCES}

Black Duck Joint Venture Management Board, 2015. The black duck joint venture: strategic plan, 2015 - 2019. pp. 37. U.S. Fish and Wildlife Service, Laurel, Maryland; Canadian Wildlife Service, Ottawa, Ontario.

Brisco, B., Touzi, R., van der Sanden, J.J., Charbonneau, F., Pultz, T.J. and D'Iorio, M., 2008. Water resource applications with RADARSAT-2-a preview. International Journal of Digital Earth, 1(1), pp.130-147.

Brisco, B., Kapfer, M., Hirose, T., Tedford, B. and Liu, J., 2011. Evaluation of C-band polarization diversity and polarimetry for wetland mapping. Canadian Journal of Remote Sensing, 37(1), pp.82-92.

Brisco, B., Li, K., Tedford, B., Charbonneau, F., Yun, S. and Murnaghan, K., 2013. Compact polarimetry assessment for rice and wetland mapping. International journal of remote sensing, 34(6), pp.1949-1964.

Brisco, B., Schmitt, A., Murnaghan, K., Kaya, S. and Roth, A., 2013. SAR polarimetric change detection for flooded vegetation. International Journal of Digital Earth, 6(2), pp.103-114.

Robertson, L.D., King, D.J. and Davies, C., 2015. Assessing land cover change and anthropogenic disturbance in wetlands using vegetation fractions derived from Landsat 5 TM imagery (19842010). Wetlands, 35(6), pp.1077-1091.

Freeman, A. and Durden, S.L., 1998. A three-component scattering model for polarimetric SAR data. IEEE Transactions on Geoscience and Remote Sensing, 36(3), pp.963-973.

Ghedira, H., Bernier, M. and Ouarda, T.B.M.J., 2000. Application of neural networks for wetland classification in RADARSAT SAR imagery. In Geoscience and Remote Sensing Symposium,
2000. Proceedings. IGARSS 2000. IEEE 2000 International (Vol. 2, pp. 675-677). IEEE.

Hess, L.L., Melack, J.M., Novo, E.M., Barbosa, C.C. and Gastil, M., 2003. Dual-season mapping of wetland inundation and vegetation for the central Amazon basin. Remote sensing of environment, 87(4), pp.404-428.

Kaya, S., Sokol, J. and Pultz, T.J., 2004. Monitoring environmental indicators of vector-borne disease from space: a new opportunity for RADARSAT-2. Canadian Journal of Remote Sensing, 30(3), pp.560-565.

Lane, C.R. and D'Amico, E., 2010. Calculating the ecosystem service of water storage in isolated wetlands using LiDAR in North Central Florida, USA. Wetlands, 30(5), pp.967-977.

Parmuchi, M.G., Karszenbaum, H. and Kandus, P., 2002. Mapping wetlands using multi-temporal RADARSAT-1 data and a decision-based classifier. Canadian Journal of Remote Sensing, 28(2), pp.175-186.

Rawat, J.S., Kumar, M.,2015. Monitoring land use/cover change using remote sensing and GIS techniques: A case study of Hawalbagh block, district Almora, Uttarakhand, India. The Egyptian Journal of Remote Sensing and Space Science, 18, pp.77-84.

Touzi R., 2007. Target scattering decomposition in terms of rollinvariant target parameters. IEEE Transactions on Geoscience and Remote Sensing. 45(1), pp.73-84.

Touzi, R., Deschamps, A. and Rother, G., 2007. Wetland characterization using polarimetric RADARSAT-2 capability. Canadian Journal of Remote Sensing, 33(sup1), pp.S56-S67.

Wdowinski, S., Kim, S.W., Amelung, F., Dixon, T.H., MirallesWilhelm, F. and Sonenshein, R., 2008. Space-based detection of wetlands' surface water level changes from L-band SAR interferometry. Remote Sensing of Environment, 112(3), pp.681696.

White, L., Brisco, B., Dabboor, M., Schmitt, A. and Pratt, A., 2015. A collection of SAR methodologies for monitoring wetlands. Remote sensing, 7(6), pp.7615-7645. 